\title{
Foreword
}

\section{Surrogate endpoints in medicine}

\author{
Sudhir Srivastava ${ }^{\mathrm{a}}$ and John A. Wagner ${ }^{\mathrm{b}}$ \\ ${ }^{a}$ Cancer Biomarkers Research Group, Division of Cancer Prevention, National Cancer Institute, 6130 Executive \\ Plaza North, Suite 3142, Bethesda, MD 20892, USA \\ ${ }^{\mathrm{b}}$ Department of Clinical Pharmacology, Merck Research Laboratories, Rahway, NJ, USA
}

A surrogate endpoint is defined as a biomarker intended to substitute for a clinical endpoint, such as an incidence, or mortality. The evidence supporting the linkage of a biomarker to accurately substitute for a clinical endpoint may be derived from epidemiologic studies, clinical trials, in vitro analysis, animal models, and simulated biologic systems. Analytical epidemiological studies of the relationship between putative intermediate endpoints and disease help postulate the nature of the interrelationship among epidemiological risk factors, intermediate endpoints, and disease. This presents problems in a system where more than one causal path exists. Adjustment for the presence of standard epidemiological factors may have different interpretations. Specifically, the disappearance of an association between a putative intermediate endpoint and disease after adjustment for the presence of some epidemiological risk factors may have opposing interpretations: either the intermediate endpoint is causal, related to disease and mediates the effects defined by the epidemiologic risk factor or it has nothing to do with disease in a causal sense.

Several contributors throughout this issue have described and used a variety of biomarkers in treatment trials. Selected biomarkers may be proposed as potential surrogate endpoints, however, relatively few are likely to achieve this status because of the complexity of disease mechanisms and the limited capability of a single biomarker to reflect the collective impact of multiple chemopreventive and therapeutic effects on outcome. It is likely that the future studies would employ a panel of biomarkers as a surrogate endpoint, which would require additional biologic studies and insights. A biomarker does not have to be causally related to a disease to play a valuable role in early detection and intervention of that disease. However, causally related biomarkers, i.e., surrogate endpoints, if measured with precision, are likely to have the strongest association with disease.

\section{Biologic characteristics of surrogate endpoints}

At least three elements are necessary to use biomarkers as surrogate outcomes: (1) the proper definition of the risk factor and how to detect it, (2) the proper description of the definitive outcome of interest and a procedure on how to assess it, and (3) knowledge of the strength and direction of the relationship between the surrogate outcome and the definitive outcome over a specified time interval. For a risk factor to be a useful surrogate outcome it must be strongly connected to the definitive outcome and the probability and direction of the relationship must be known. Surrogate outcomes do not shorten the first investigation because the relationship between the risk factor and the true outcome must be known prior to using the risk factor as a surrogate outcome.

Several basic criteria must be met before the potential markers could serve as adequate surrogate endpoints either for risk or clinical outcome: (1) Is the surrogate biomarker differentially expressed in normal and high-risk tumor tissues? (2) At what stage of carcinogenesis does the marker appear? (3) Does the marker, and its assay, provide acceptable sensitivity, specificity and accuracy, and (4) How easily can the marker be measured? For surrogate biomarkers to serve as intermediate endpoints, it would be desirable to satisfy additional criteria: (1) Can the marker be modulated by chemopreventive agents? and (2) Does modulation of the marker correlate with a change in disease rate? 


\section{Laboratory characteristics of surrogate endpoints}

A surrogate endpoint or biomarker must be quantifiable in an appropriate biologic specimen and indicative of pharmacologic response to a chemopreventive or therapeutic agents. These must meet the following criteria: (1) the biomarker must be stable, and in case of adducts as biomarkers, must have a longer half-life, (2) the assay for biomarkers must be reproducible and must have high sensitivity and specificity, (3) must have minimum diurnal variations and (4) the modulation preferably be consistent, at least with the same class of the chemopreventive or therapeutic agents.

\section{Interventional assessment of surrogate endpoints}

The surrogate endpoint must be assessed for its efficacy. The recently established NCI Early Detec- tion Research Network (http://www.cancer.gov/edrn) has undertaken a major step in developing and validating biomarkers for evaluating cancer risk and detecting early cancer. The Network links centers of expertise in tumor biology, diagnostic technologies, and clinicaltrials methodology in academia and industry to develop high-throughput assays suitable for clinical application. These laboratories/centers cover a range of study designs, technology developments, and innovative approaches from genomics to proteomics in pursuit of developing molecular, genetic and biologic markers for earlier cancer detection and identification of high-risk subjects, which will provide powerful tools for identifying suitable cohorts for chemoprevention studies in the future.

Finally, the special issue of Disease Markers "Surrogate Endpoints in Medicine" provides a valuable resource for researchers engaged in clinical trials. 


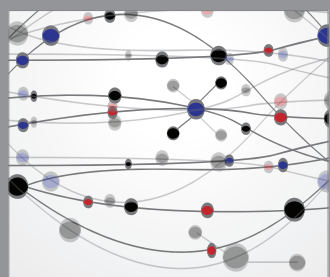

The Scientific World Journal
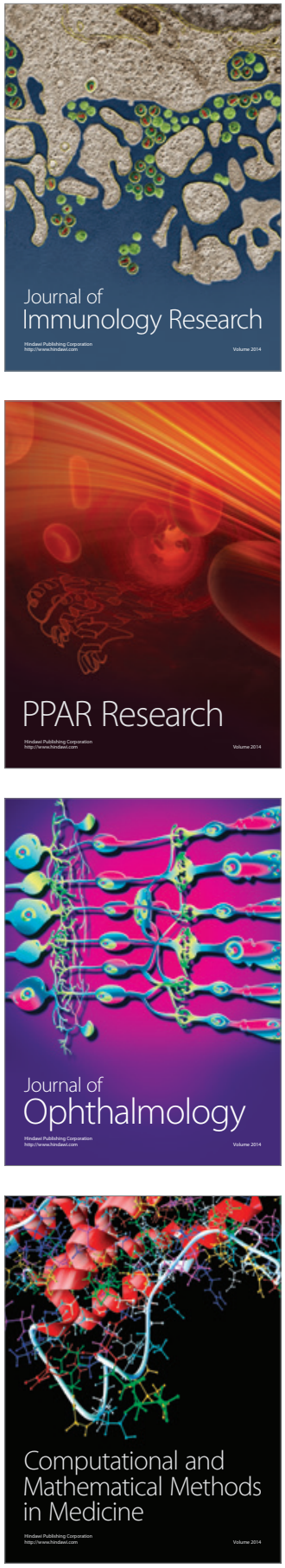

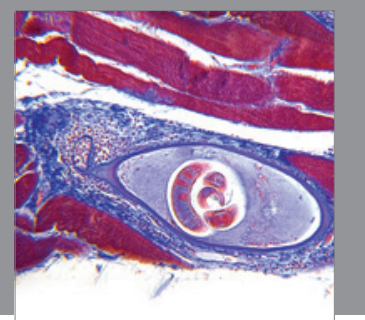

Gastroenterology

Research and Practice
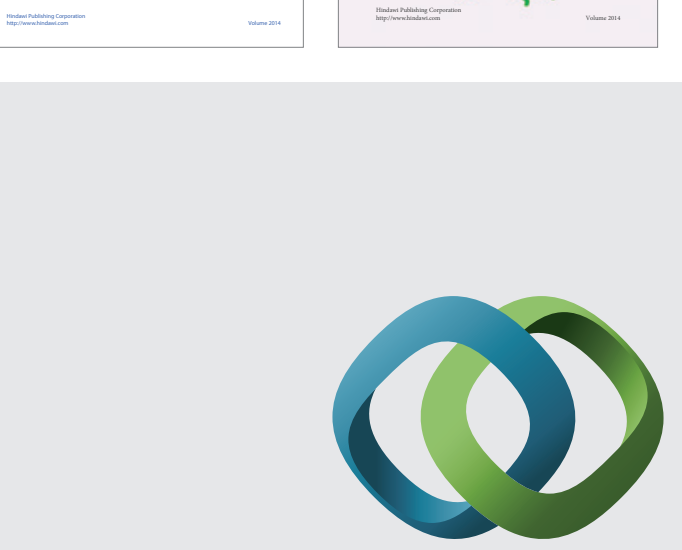

\section{Hindawi}

Submit your manuscripts at

http://www.hindawi.com
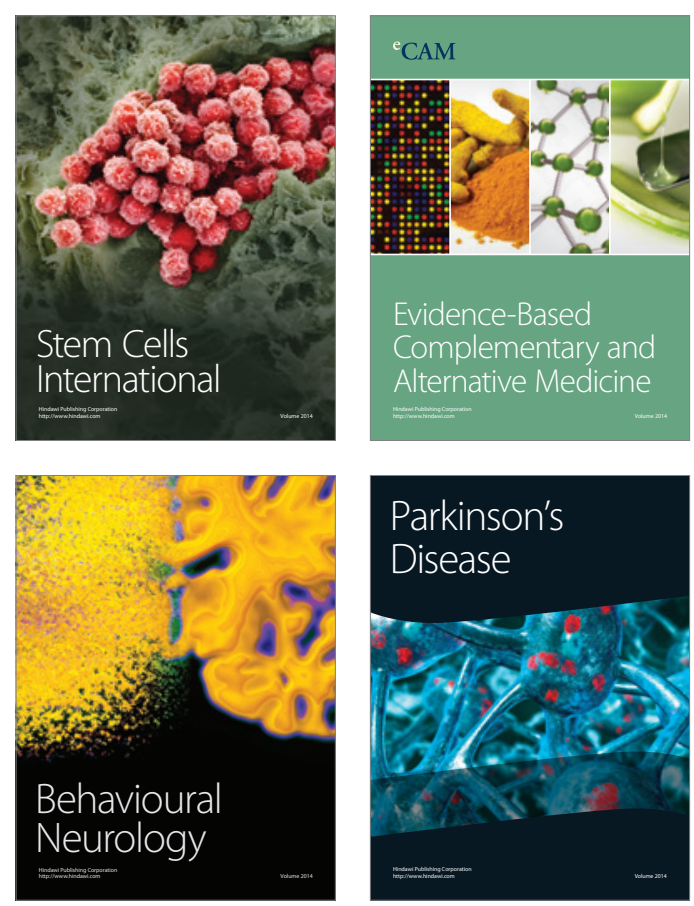

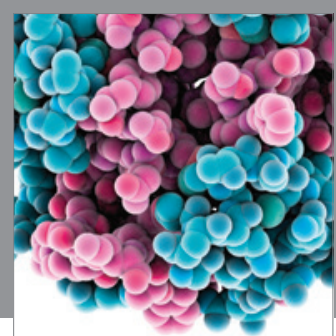

Journal of
Diabetes Research

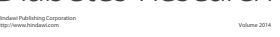

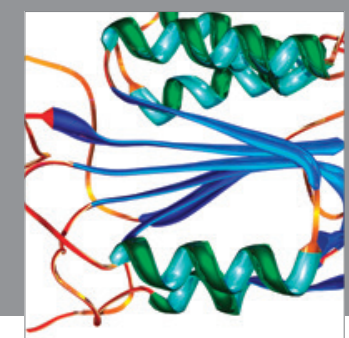

Disease Markers
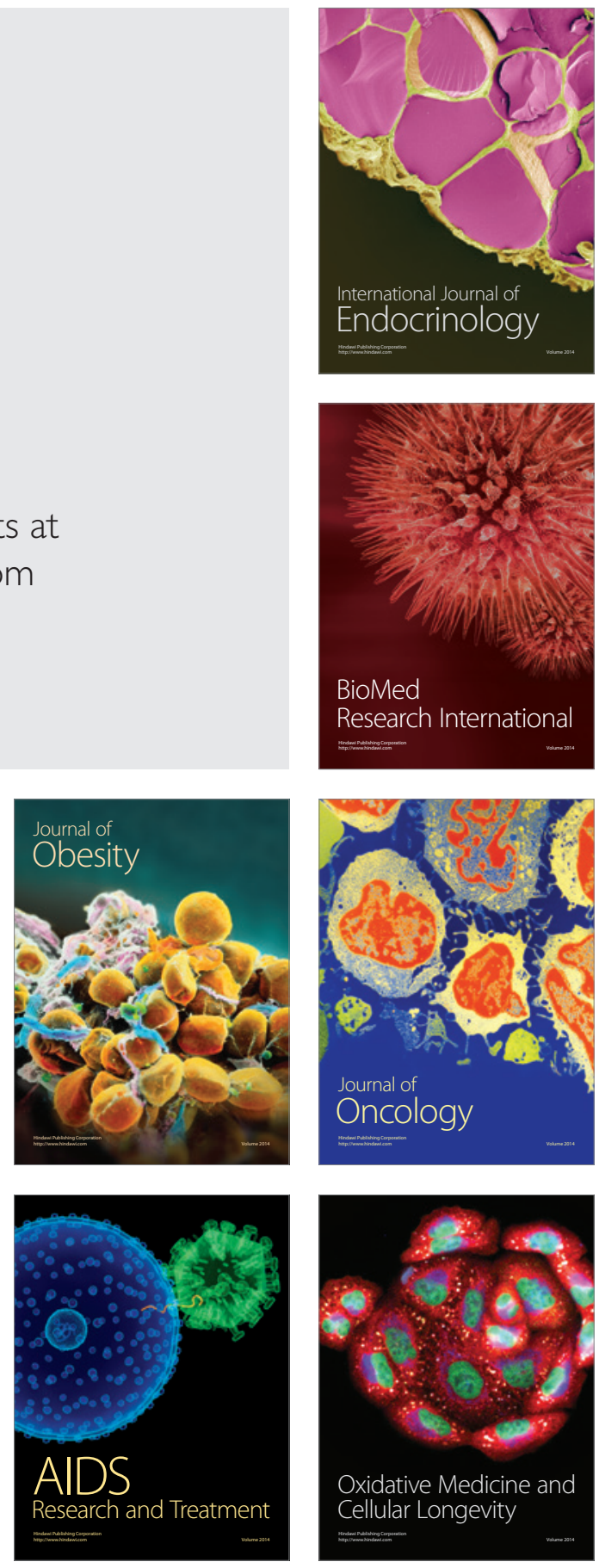